\title{
First limits on the very-high energy gamma-ray afterglow emission of a fast radio burst
}

\section{H.E.S.S. observations of FRB 150418}

H.E.S.S. Collaboration, H. Abdalla ${ }^{1}$, A. Abramowski ${ }^{2}$, F. Aharonian ${ }^{3,4,5}$, F. Ait Benkhali ${ }^{3}$, A. G. Akhperjanian ${ }^{\dagger, 6,5}$, T. Andersson $^{10}$, E. O. Angüner ${ }^{21}$, M. Arakawa ${ }^{41}$, M. Arrieta ${ }^{15}$, P. Aubert ${ }^{23}$, M. Backes ${ }^{8}$, A. Balzer ${ }^{9}$, M. Barnard ${ }^{1}$, Y. Becherini ${ }^{10}$, J. Becker Tjus ${ }^{11}$, D. Berge $^{12}$, S. Bernhard ${ }^{13}$, K. Bernlöhr ${ }^{3}$, R. Blackwell ${ }^{14}$, M. Böttcher ${ }^{1}$, C. Boisson ${ }^{15}$, J. Bolmont ${ }^{16}$, P. Bordas ${ }^{3}$, J. Bregeon ${ }^{17}$, F. Brun ${ }^{25}$, P. Brun ${ }^{18}$, M. Bryan ${ }^{9}$, M. Büchele ${ }^{34}$, T. Bulik ${ }^{19}$, M. Capasso ${ }^{27}$, J. Carr ${ }^{20}$, S. Casanova ${ }^{21,3}$, M. Cerruti ${ }^{16}$, N. Chakraborty ${ }^{3}$, R. Chalme-Calvet ${ }^{16}$, R. C. G. Chaves ${ }^{17}$, A. Chen ${ }^{22}$, J. Chevalier ${ }^{23}$, M. Chrétien ${ }^{16}$, M. Coffaro ${ }^{27}$, S. Colafrancesco ${ }^{22}$, G. Cologna ${ }^{24}$, B. Condon ${ }^{25}$, J. Conrad ${ }^{26}$, Y. Cui ${ }^{27}$, I. D. Davids ${ }^{1,8}$, J. Decock ${ }^{18}$, B. Degrange ${ }^{28}$, C. Deil ${ }^{3}$, J. Devin ${ }^{17}$, P. deWilt ${ }^{14}$, L. Dirson ${ }^{2}$, A. Djannati-Atai ${ }^{29}$, W. Domainko ${ }^{3}$, A. Donath ${ }^{3}$, L. O'C. Drury ${ }^{4}$,

K. Dutson ${ }^{31}$, J. Dyks ${ }^{32}$, T. Edwards ${ }^{3}$, K. Egberts ${ }^{33}$, P. Eger ${ }^{3}$, J.-P. Ernenwein ${ }^{20}$, S. Eschbach ${ }^{34}$, C. Farnier ${ }^{26,10}$, S. Fegan ${ }^{28}$, M. V. Fernandes ${ }^{2}$,

A. Fiasson ${ }^{23}$, G. Fontaine ${ }^{28}$, A. Förster ${ }^{3}$, S. Funk ${ }^{34}$, M. Füßling ${ }^{35}$, S. Gabici ${ }^{29}$, M. Gajdus ${ }^{7}$, Y. A. Gallant ${ }^{17}$, T. Garrigoux ${ }^{1}$, G. Giavitto ${ }^{35}$,

B. Giebels ${ }^{28}$, J. F. Glicenstein ${ }^{18}$, D. Gottschall ${ }^{27}$, A. Goyal ${ }^{36}$, M.-H. Grondin ${ }^{25}$, J. Hahn ${ }^{3}$, M. Haupt ${ }^{35}$, J. Hawkes ${ }^{14}$, G. Heinzelmann ${ }^{2}$, G. Henri ${ }^{30}$, G. Hermann ${ }^{3}$, O. Hervet ${ }^{15,46}$, J. A. Hinton ${ }^{3}$, W. Hofmann ${ }^{3}$, C. Hoischen ${ }^{33}$, M. Holler $^{28}$, D. Horns ${ }^{2}$, A. Ivascenko ${ }^{1}$, H. Iwasaki ${ }^{41}$,

A. Jacholkowska ${ }^{16}$, M. Jamrozy ${ }^{36}$, M. Janiak ${ }^{32}$, D. Jankowsky ${ }^{34}$, F. Jankowsky ${ }^{24}$, M. Jingo ${ }^{22}$, T. Jogler ${ }^{34}$, L. Jouvin ${ }^{29}$, I. Jung-Richardt ${ }^{34}$,

M. A. Kastendieck ${ }^{2}$, K. Katarzyński ${ }^{37}$, M. Katsuragawa ${ }^{42}$, U. Katz ${ }^{34}$, D. Kerszberg ${ }^{16}$, D. Khangulyan ${ }^{41}$, B. Khélifi' ${ }^{29}$, M. Kieffer ${ }^{16}$, J. King ${ }^{3, \star}$,

S. Klepser ${ }^{35}$, D. Klochkov ${ }^{27}$, W. Kluźniak ${ }^{32}$, D. Kolitzus ${ }^{13}$, Nu. Komin ${ }^{22}$, K. Kosack ${ }^{18}$, S. Krakau ${ }^{11}$, M. Kraus ${ }^{34}$, P. P. Krüger ${ }^{1}$, H. Laffon ${ }^{25}$,

G. Lamanna ${ }^{23}$, J. Lau ${ }^{14}$, J.-P. Lees ${ }^{23}$, J. Lefaucheur ${ }^{15}$, V. Lefranc ${ }^{18}$, A. Lemière ${ }^{29}$, M. Lemoine-Goumard ${ }^{25}$, J.-P. Lenain ${ }^{16}$, E. Leser ${ }^{33}$, T. Lohse ${ }^{7}$, M. Lorentz ${ }^{18}$, R. Liu ${ }^{3}$, R. López-Coto ${ }^{3}$, I. Lypova ${ }^{35}$, V. Marandon ${ }^{3}$, A. Marcowith ${ }^{17}$, C. Mariaud ${ }^{28}$, R. Marx ${ }^{3}$, G. Maurin ${ }^{23}$, N. Maxted ${ }^{14}$,

M. Mayer ${ }^{7}$, P. J. Meintjes ${ }^{38}$, M. Meyer ${ }^{26}$, A. M. W. Mitchell ${ }^{3}$, R. Moderski ${ }^{32}$, M. Mohamed $^{24}$, L. Mohrmann ${ }^{34}$, K. Morä $^{26}$, E. Moulin ${ }^{18}$,

T. Murach ${ }^{7}$, S. Nakashima ${ }^{42}$, M. de Naurois ${ }^{28}$, F. Niederwanger ${ }^{13}$, J. Niemiec ${ }^{20}$, L. Oakes ${ }^{7}$, P. O’ Brien $^{31}$, H. Odaka ${ }^{42}$, S. Öttl ${ }^{13}$, S. Ohm ${ }^{35}$,

M. Ostrowski ${ }^{36}$, I. Oya ${ }^{35}$, M. Padovani ${ }^{17}$, M. Panter ${ }^{3}$, R. D. Parsons ${ }^{3}$, N. W. Pekeur ${ }^{1}$, G. Pelletier ${ }^{30}$, C. Perennes ${ }^{16}$, P.-O. Petrucci ${ }^{30}$, B. Peyaud $^{18}$,

Q. Piel ${ }^{23}$, S. Pita ${ }^{29}$, H. Poon ${ }^{3}$, D. Prokhorov ${ }^{10}$, H. Prokoph ${ }^{10}$, G. Pühlhofer ${ }^{27}$, M. Punch ${ }^{29,10}$, A. Quirrenbach ${ }^{24}$, S. Raab ${ }^{34}$, A. Reimer ${ }^{13}$,

O. Reimer ${ }^{13}$, M. Renaud ${ }^{17}$, R. de los Reyes ${ }^{3}$, S. Richter ${ }^{1}$, F. Rieger ${ }^{3,39}$, C. Romoli ${ }^{4}$, G. Rowell ${ }^{14, \star}$, B. Rudak ${ }^{32}$, C. B. Rulten ${ }^{15}$, V. Sahakian ${ }^{6,5}$, S. Saito ${ }^{41}$, D. Salek ${ }^{40}$, D. A. Sanchez ${ }^{23}$, A. Santangelo ${ }^{27}$, M. Sasaki ${ }^{27}$, R. Schlickeiser ${ }^{11}$, F. Schüssler ${ }^{18, \star}$, A. Schulz ${ }^{35}$, U. Schwanke ${ }^{7}$,

S. Schwemmer ${ }^{24}$, M. Seglar-Arroyo ${ }^{18}$, M. Settimo ${ }^{16}$, A. S. Seyffert ${ }^{1}$, N. Shafi' ${ }^{22}$, I. Shilon ${ }^{34}$, R. Simoni ${ }^{9}$, H. Sol ${ }^{15}$, F. Spanier ${ }^{1}$, G. Spengler ${ }^{26}$, F. Spies ${ }^{2}$, Ł. Stawarz ${ }^{36}$, R. Steenkamp ${ }^{8}$, C. Stegmann ${ }^{33,35}$, K. Stycz ${ }^{35}$, I. Sushch ${ }^{1}$, T. Takahashi2 ${ }^{42}$, J.-P. Tavernet ${ }^{16}$, T. Tavernier ${ }^{29}$, A. M. Taylor ${ }^{4}$, R. Terrier ${ }^{29}$, L. Tibaldo ${ }^{3}$, D. Tiziani ${ }^{34}$, M. Tluczykont ${ }^{2}$, C. Trichard ${ }^{20}$, N. Tsuji ${ }^{41}$, R. Tuffs ${ }^{3}$, Y. Uchiyama ${ }^{41}$, D. J. van der Walt ${ }^{1}$, C. van Eldik ${ }^{34}$, C. van Rensburg ${ }^{1}$, B. van Soelen ${ }^{38}$, G. Vasileiadis ${ }^{17}$, J. Veh $^{34}$, C. Venter ${ }^{1}$, A. Viana ${ }^{3}$, P. Vincent ${ }^{16}$, J. Vink ${ }^{9}$, F. Voisin ${ }^{14}$, H. J. Völk ${ }^{3}$,

T. Vuillaume ${ }^{23}$, Z. Wadiasingh ${ }^{1}$, S. J. Wagner ${ }^{24}$, P. Wagner ${ }^{7}$, R. M. Wagner ${ }^{26}$, R. White ${ }^{3}$, A. Wierzcholska ${ }^{21}$, P. Willmann $^{34}$, A. Wörnlein ${ }^{34}$,

D. Wouters ${ }^{18}$, R. Yang ${ }^{3}$, V. Zabalza ${ }^{31}$, D. Zaborov ${ }^{28}$, M. Zacharias ${ }^{24}$, R. Zanin ${ }^{3}$, A. A. Zdziarski ${ }^{32}$, A. Zech ${ }^{15}$, F. Zefi $^{28}$, A. Ziegler $^{34}$, N. Żywucka ${ }^{36}$, SUPERB Collaboration, F. Jankowski ${ }^{43}$, E. F. Keane ${ }^{44}$, and E. Petroff ${ }^{12,45}$

(Affiliations can be found after the references)

Received 14 June 2016 / Accepted 15 November 2016

\section{ABSTRACT}

Aims. Following the detection of the fast radio burst FRB150418 by the SUPERB project at the Parkes radio telescope, we aim to search for very-high energy gamma-ray afterglow emission.

Methods. Follow-up observations in the very-high energy gamma-ray domain were obtained with the H.E.S.S. imaging atmospheric Cherenkov telescope system within $14.5 \mathrm{~h}$ of the radio burst.

Results. The obtained $1.4 \mathrm{~h}$ of gamma-ray observations are presented and discussed. At the $99 \%$ C.L. we obtained an integral upper limit on the gamma-ray flux of $\Phi_{\gamma}(E>350 \mathrm{GeV})<1.33 \times 10^{-8} \mathrm{~m}^{-2} \mathrm{~s}^{-1}$. Differential flux upper limits as function of the photon energy were derived and used to constrain the intrinsic high-energy afterglow emission of FRB 150418.

Conclusions. No hints for high-energy afterglow emission of FRB 150418 were found. Taking absorption on the extragalactic background light into account and assuming a distance of $z=0.492$ based on radio and optical counterpart studies and consistent with the FRB dispersion, we constrain the gamma-ray luminosity at $1 \mathrm{TeV}$ to $L<5.1 \times 10^{47} \mathrm{erg} / \mathrm{s}$ at $99 \%$ C.L.

Key words. gamma rays: general - astroparticle physics

\section{Introduction}

Fast radio bursts (FRBs) are one of the major astronomical mysteries that have emerged in the last decade. First noticed in 2007 in archival data taken with the Parkes radio telescope (Lorimer et al. 2007), seventeen of these millisecondduration bursts have been detected so far (Thornton et al. 2013; Petroff et al. 2015). The majority were found with the Parkes

\footnotetext{
$\dagger$ Deceased.

* Corresponding authors.

e-mail: contact.hess@hess-experiment.eu
}

telescopes, although additional bursts have been detected with the Arecibo telescope (Spitler et al. 2014) and the Green Bank Telescope (GBT; Masui et al. 2015). A summary of known FRBs including the details of the observations can be found in the online catalog FRBCAT $^{1}$ (Petroff et al. 2016).

The frequency-dependent dispersion properties of FRBs have constrained their distance to $z \sim 0.1-1$ (Petroff et al. 2016). Distance confusion can, however, arise due to the unknown plasma density within the supposed host galaxy of the FRB, and

\footnotetext{
http://wWw . astronomy. swin. edu. au/frbcat/
} 
that of our own Galaxy (the latter is especially relevant in cases where the FRB was observed toward the Galactic plane).

The typical radio energy output of a few $10^{39} D_{1 \mathrm{Gpc}}^{2} \mathrm{erg}$, assuming isotropic emission at distance $D_{1 \mathrm{Gpc}}=D / 1 \mathrm{Gpc}$, and the millisecond duration of FRBs have led to proposed scenarios involving compact objects - white dwarfs (WDs), neutron stars (NSs) and/or black holes (BHs). A review of potential sources can be found for example in Kulkarni et al. (2014). The merger of various combinations of WDs, NSs and/or BHs are generally favoured (e.g., Totani 2013; Zhang 2014; Kashiyama et al. 2013; Mingarelli et al. 2015) in what would be a cataclysmic event similar to short gamma-ray bursts (sGRBs). Other models involve young pulsars created in core-collapse supernovae of massive stars (Connor et al. 2016) and blitzars (BH forming rapidly from a NS via accretion, Falcke \& Rezzolla 2014). The recent discovery of repeating bursts from FRB121102 (Spitler et al. 2016; Scholz et al. 2016) has renewed attention in non-cataclysmic scenarios such as flares and giant pulses from NSs and/or magnetars (Lyubarsky 2014; Katz 2016; Pen \& Connor 2015; Cordes \& Wasserman 2016).

A potentially significant advance in our understanding of FRBs came with the detection of a radio afterglow at the location of FRB150418 with the Australia Telescope Compact Array (ATCA, Keane et al. 2016). The burst FRB150418 was initially detected at Parkes on the 18th April 2015 by the SUPERB team. The fading radio afterglow lasted up to six days after the FRB, and could be linked to an elliptical host galaxy at $z=0.492 \pm 0.008$ (WISE J071634.59-190039.2). If connected to the afterglow, the energetics of FRB150418 suggest a cataclysmic origin of the bursts (e.g. Zhang 2016). However, alternative explanations for the temporal behavior of the radio flux have been suggested in the form of an unrelated active galactic nucleus (AGN) activity in the host galaxy (Williams \& Berger 2016), or interstellar scintillation (Akiyama \& Johnson 2016). Several other possible scenarios could also explain the ATCA source, including an AGN related to the FRB (Vedantham et al. 2016), a magnetar (so the FRB repeats at the same dispersion measure as FRB150418), localized star formation, a long GRB afterglow (as seen in GRB130925A, Horesh et al. 2015), or a yet unknown mechanism. Ongoing radio monitoring may resolve the issue in the future.

FRBs release enormous amounts of energy in the radio domain (e.g., FRB150418 released $8_{-5}^{+1} \times 10^{38} \mathrm{erg}$ at the position of the potential host galaxy with a luminosity greater than $1.3 \times 10^{42} \mathrm{erg} / \mathrm{s}$ ) and their potential origins are thought to be similar to other transients seen in the X-ray and multi-GeV gammaray bands such as short and/or long GRBs (Zhang 2014). Several FRB models have also specifically suggested the existence of flares in the TeV band (e.g., Lyubarsky 2014; Murase et al. 2016) and proposed follow-ups of FRBs at very high energies.

In this paper, we report the first follow-up observations of FRBs in very high energy (VHE) gamma-rays of TeV $\left(10^{12} \mathrm{eV}\right)$ energies. We present observations searching for the very highenergy afterglow of FRB150418 with the High Energy Stereoscopic System (H.E.S.S.) following an alert from the SUPERB collaboration.

\section{Observations from H.E.S.S. and data analysis}

Dedicated follow-up observations of FRB150418 were obtained in the very-high energy gamma-ray domain with the H.E.S.S. imaging atmospheric Cherenkov telescope array. H.E.S.S. is located on the Khomas Highland plateau of Namibia $\left(23^{\circ} 16^{\prime} 18^{\prime \prime}\right.$ South, $16^{\circ} 30^{\prime} 00^{\prime \prime}$ East), at an elevation of $1800 \mathrm{~m}$ above sea

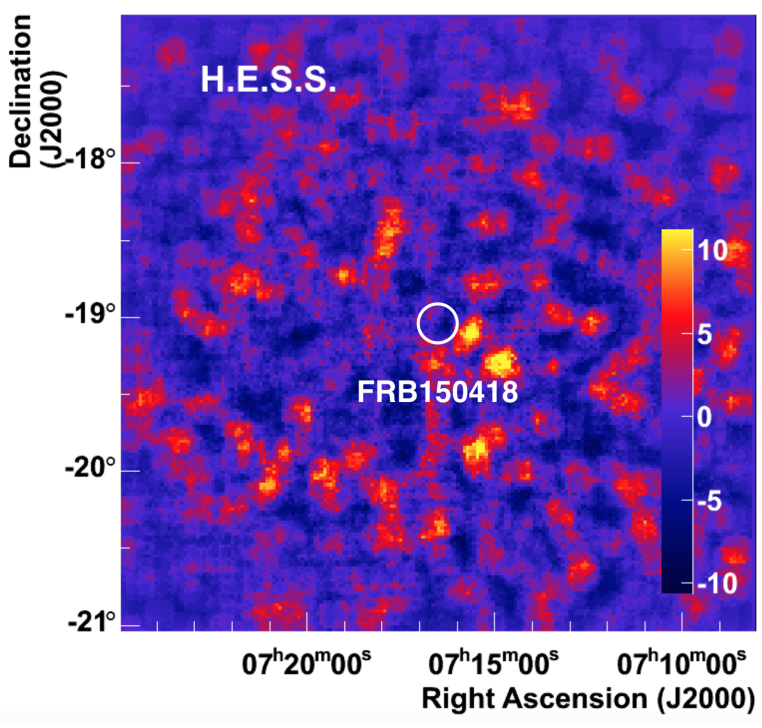

Fig. 1. VHE gamma-ray emission around the direction of FRB150418 illustrated by the event counts exceeding the background. The circle in the center has a diameter of $0.24^{\circ}$ and denotes the width of the Parkes beam in which the burst has been observed.

level. With its original four-telescope array, H.E.S.S. is sensitive to cosmic and gamma-rays in the $100 \mathrm{GeV}$ to $100 \mathrm{TeV}$ energy range and is capable of detecting a Crab-like source close to zenith and under good observational conditions at the $5 \sigma$ level within less than one minute (Aharonian et al. 2006). In 2012 a fifth telescope with $28 \mathrm{~m}$ diameter was commissioned, extending the covered energy range toward lower energies. This fifth telescope was unavailable at the time of the observation and data for the follow-up presented here have therefore been obtained with the four $12 \mathrm{~m}$ H.E.S.S. telescopes.

The notification of FRB150418 was received from the SUPERB team on 2015-04-18 during daytime at the site of the H.E.S.S. experiment, thus prohibiting prompt follow-up observations. The necessary observation conditions were reached the evening of the same day at 17:55 UTC (about 14.5 h after the FRB) and $1.4 \mathrm{~h}$ of data could be recorded until the source set below an elevation of $45^{\circ}$, which is the typical horizon for observations retaining a relatively low energy threshold. The data, taken in standard wobble mode operations with source offsets of $0.7^{\circ}$, fulfill all standard data quality criteria including requirements on atmospheric conditions, and detector stability. The zenith angle of the observations ranged from $21^{\circ}$ to $42^{\circ}$. After correcting for acceptance effects due to the wobble source offsets, a total effective live-time of $1.1 \mathrm{~h}$ at the FRB position was available for analysis.

The data were analyzed using Model Analysis (de Naurois \& Rolland 2009), an advanced Cherenkov image reconstruction method in which the recorded shower images of all triggered telescopes are compared to a semi-analytical model of gamma ray showers by means of a log-likelihood optimization. The "standard cuts" of Model Analysis were adopted. These cuts require, among other criteria, the total charge in the shower image to be greater than 60 photoelectrons. The resulting energy threshold, defined as the energy where the acceptance is $20 \%$ of its maximum value, is $350 \mathrm{GeV}$ for this dataset.

The robustness and stability of the described analysis have been verified with an independent analysis relying on an independent data calibration chain and using the Image Pixel-wise fit for Atmospheric Cherenkov Telescopes (ImPACT, Parsons \& Hinton 2014) reconstruction method. The results 
H.E.S.S. Collaboration et al.: First limits on the very-high energy gamma-ray afterglow emission of a fast radio burst
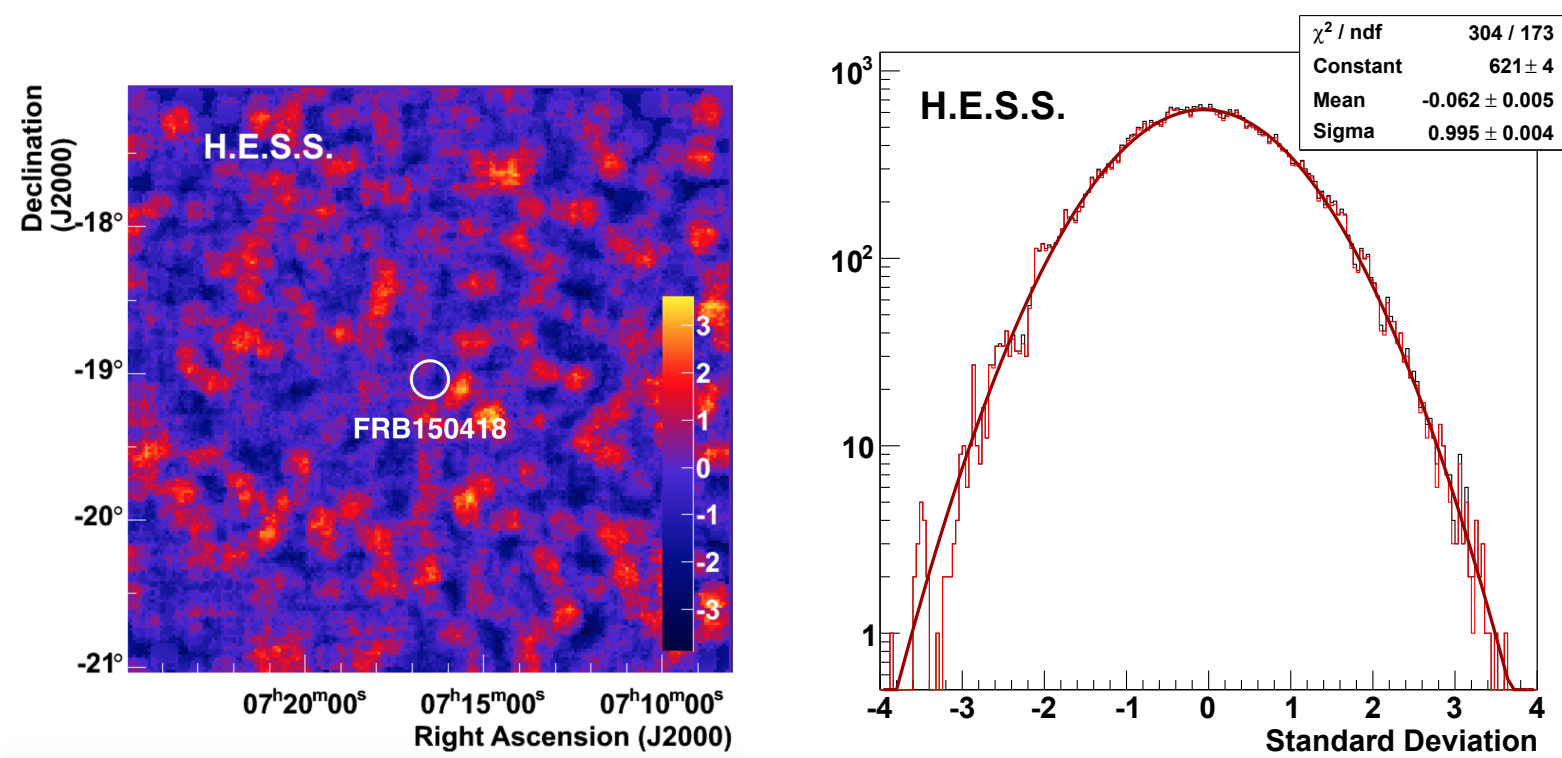

Fig. 2. Left plot: map of significances of the gamma ray emission using the formalism proposed by Li \& Ma (1983) in the region around FRB150418. The circle in the center has a diameter of $0.24^{\circ}$ and denotes the width of the Parkes beam in which the burst was observed. Right plot: distribution of significances (black histogram) compared to the distribution obtained by excluding a circular region of $0.25^{\circ}$ radius (red histogram). The red line and the shown parameters correspond to a Gaussian function fit to the latter distribution.

from this cross-check analysis are consistent with the ones presented here.

The H.E.S.S. field-of-view (FoV) with a diameter of $5^{\circ}$ easily covers the Parkes beam with a FWHM of $0.24^{\circ}$ (Keane et al. 2016). The H.E.S.S. observations therefore cover all potential locations of FRB150418 within the Parkes beam in which the FRB was detected. On the other hand, the H.E.S.S. point-spread function has a diameter of $\sim 0.12^{\circ}(68 \%$ containment $)$, that is, half the Parkes beam size. We can therefore, based solely on H.E.S.S. data, not expect to easily resolve the origin of a potential afterglow within the Parkes beam and would not be able to discriminate between the potential host galaxy discussed by Keane et al. (2016) and other locations within the beam.

No high-energy gamma ray source has been detected within the region of interest in the four-year long observations by the LAT instrument onboard the Fermi satellite (Acero et al. 2015). Also no emission at very-high gamma-ray energies has been reported so far from the region ${ }^{2}$.

The background level in the FoV was determined from the dataset itself using the standard "ring background" technique (Berge et al. 2007), a robust method ideally suited to deriving gamma-ray emission maps in FoVs with low numbers of sources. In order to derive the acceptance function required as input to the ring background method we exploited the azimuthal symmetry of the acceptance across the field-of-view of the telescopes. We derive the acceptance from the same dataset and, in order to reduce systematic uncertainties due to the limited statistics, we refrained from a detailed modeling of the zenith angle dependence of the acceptance function and use the acceptance derived at the average zenith angle of $32^{\circ}$.

\section{Results}

The map of gamma-ray events exceeding the background is shown for the full region of interest (ROI) around FRB150418 in Fig. 1. We then converted the excess counts into significance levels using the formalism described by Li \& Ma (1983). The resulting map of significances is shown in the left plot of Fig. 2.

\footnotetext{
2 http://tevcat.uchicago.edu
}

It should be noted that trial factors due to the large number of individual bins are not accounted for in this representation. For an ROI dominated by statistical fluctuations of the background the distribution of the significances should follow a Gaussian with a mean at zero and a width of one. The right plot in Fig. 2 shows the corresponding distribution (black histogram). The distribution obtained by excluding a circular "signal" region of $0.25^{\circ}$ radius around the FRB position is shown in red. Both histograms agree very well. In addition, when fitting the latter distribution with a Gaussian shape, very good agreement with the "background only" hypothesis was found. It can be noted that the errors on the obtained parameters are underestimated due to correlations in the entries of the significance distributions which are introduced by the background estimation on overlapping regions. We conclude that the ROI is well described and clearly dominated by background events.

As the obtained results were fully compatible with the background expectation we conclude that no significant gamma-ray afterglow was detected from the direction of FRB150418 (cf. Fig. 2). Consequently we derive $99 \%$ C.L. upper limits on the gamma-ray flux as function of energy following the approach by Feldman \& Cousins (1998). Assuming a generic $E^{-2}$ energy spectrum for the potential emission and integrating above the threshold of $350 \mathrm{GeV}$ we obtain $\Phi_{\gamma}(E>350 \mathrm{GeV})<$ $1.33 \times 10^{-8} \mathrm{~m}^{-2} \mathrm{~s}^{-1}$. Assuming a $E^{-4}$ energy spectrum, we obtain $\Phi_{\gamma}(E>350 \mathrm{GeV})<2.12 \times 10^{-8} \mathrm{~m}^{-2} \mathrm{~s}^{-1}$. Differential upper limits as function of the energy are shown as black arrows in Fig. 3. Due to the small size of the bins, the influence of the assumed spectrum (e.g. $E^{-2}$ vs. $\left.E^{-4}\right)$ on the differential upper limits is less than $1.3 \%$.

While propagating through the extragalactic radiation fields, high-energy gamma rays interact with the extragalactic background light (EBL) via $\mathrm{e}^{+} / \mathrm{e}^{-}$pair-creation processes. This leads to the collective effect of an absorption of gamma-rays at the highest energies. The resulting gamma-ray opacity depends on the energy as well as on the distance of the source. Using the EBL model published in Gilmore et al. (2012) we were able to correct the derived upper limits on the gamma-ray flux measured on Earth for EBL absorption effects and thus derive 


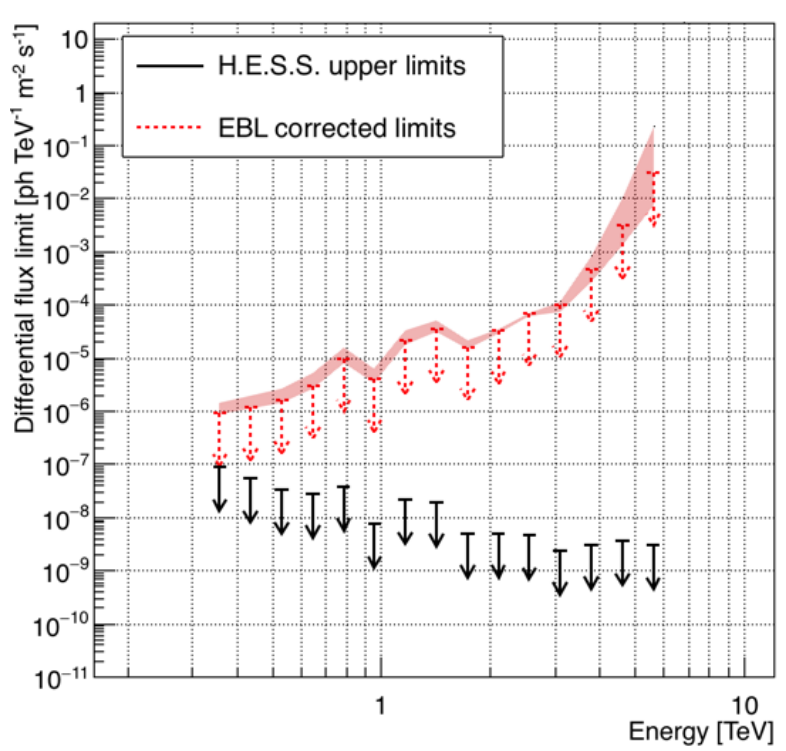

Fig. 3. Limits ( $99 \% \mathrm{CL}$ ) on the very high energy gamma-ray flux derived from the H.E.S.S. follow-up on FRB150418 assuming an $E^{-2}$ energy spectrum. The EBL de-absorption is based on the model from Gilmore et al. (2012) and assumes the FRB distance of $z=$ 0.492 (Keane et al. 2016). The uncertainty induced by different EBL models is shown as red band.

energy dependent intrinsic flux limits of the FRB. The result shown in Fig. 3 has been derived using the redshift of the potential host galaxy of FRB150418, $z=0.492$ (Keane et al. 2016). While this distance is consistent with the one derived from the dispersion measure of the FRB, there is still controversy as to the relationship between FRB150418 and WISE J071634.59-190039.2. The intrinsic limits shown should therefore not be taken as definitive, but rather as an illustration of how the EBL absorption impacts the constraints as a function of energy. The red band in Fig. 3 illustrates the effect of different EBL models (e.g. Gilmore et al. 2012; Franceschini et al. 2008; Dominguez et al. 2011) on the EBL correction of the derived flux limits.

\section{Discussion and conclusion}

We have reported the first follow-up observations of fast radio bursts in the very high-energy gamma-ray domain. The origin of FRBs remains elusive, and observational constraints such as those presented here are crucial pieces for solving this puzzle. In addition to an enlarged wavelength coverage, timely observations are essential in order to be able to cover as many of the potentially very rapid emission scenarios as possible.

The luminosity in the radio domain of FRB150408 has been estimated to $L>1.3 \times 10^{42} \mathrm{erg} / \mathrm{s}$ (Keane et al. 2016). The first non-radio observations of the emission region of FRB150418 were carried out $8 \mathrm{~h}$ after the radio burst by the Swift Xray satellite and a $3 \sigma$ upper limit on the X-ray flux of $\Phi_{\mathrm{X}}<$ $7.1 \times 10^{-14} \mathrm{erg} \mathrm{cm}^{-2} \mathrm{~s}^{-1}$ has been derived (Keane et al. 2016). Our VHE gamma-ray observations constrain emission at slightly longer timescales (starting $14.5 \mathrm{~h}$ after the burst, due to the inability of Imaging Atmospheric Cherenkov Telescopes to observe during daytime) and provide valuable input to models specifically suggesting flares in the TeV band (Lyubarsky 2014; Murase et al. 2016). Taking absorption on the extragalactic background light into account, as shown in Fig. 3, and assuming a distance of $z=0.492$ based on radio and optical counterpart studies and consistent with the FRB dispersion, we constrain the gamma-ray luminosity of the afterglow of FRB150418 at $1 \mathrm{TeV}$ to $L<5.1 \times 10^{47} \mathrm{erg} / \mathrm{s}$ at $99 \%$ C.L.

Acknowledgements. The support of the Namibian authorities and of the University of Namibia in facilitating the construction and operation of H.E.S.S. is gratefully acknowledged, as is the support by the German Ministry for Education and Research (BMBF), the Max Planck Society, the German Research Foundation (DFG), the French Ministry for Research, the CNRS-IN2P3 and the Astroparticle Interdisciplinary Programme of the CNRS, the UK Science and Technology Facilities Council (STFC), the IPNP of the Charles University, the Czech Science Foundation, the Polish Ministry of Science and Higher Education, the South African Department of Science and Technology and National Research Foundation, the University of Namibia, the Innsbruck University, the Austrian Science Fund (FWF), and the Austrian Federal Ministry for Science, Research and Economy, and by the University of Adelaide and the Australian Research Council. We appreciate the excellent work of the technical support staff in Berlin, Durham, Hamburg, Heidelberg, Palaiseau, Paris, Saclay, and in Namibia in the construction and operation of the equipment. This work benefited from services provided by the H.E.S.S. Virtual Organisation, supported by the national resource providers of the EGI Federation.

\section{References}

Acero, F., Ackermann, M., Ajello, M., et al. 2015, ApJ, 218, 23

Aharonian, F., Akhperjanian, A. G., Bazer-Bachi, A. R., et al. 2006, A\&A, 457, 899

Akiyama, K., \& Johnson, M. D. 2016, ApJ, 824, L3

Berge, D., Funk, S., \& Hinton, J. 2007, A\&A, 466, 1219

Connor, L., Sievers, J., \& Pen, U.-L. 2016, MNRAS, 458, L19

Cordes, J. M., \& Wasserman, I. 2016, MNRAS, 457, 232

de Naurois, M., \& Rolland, L. 2009, Astropart. Phys., 32, 231

Dominguez, A., Primack, J. R., Rosario, D. J., et al. 2011, MNRAS, 410, 2556

Falcke, H., \& Rezzolla, L. 2014, A\&A, 562, A137

Feldman, G. J., \& Cousins, R. D. 1998, Phys. Rev. D, 57, 3873

Franceschini, A., Rodighiero, G., \& Vaccari, M. 2008, A\&A, 487, 837

Gilmore, R. C., Somerville, R. S., Primack, J. R., \& Dominguez, A. 2012, MNRAS, 422, 3189

Horesh, A., Cenko, S. B., Perley, D. A., et al. 2015, ApJ, 812, 86

Kashiyama, K., Ioka, K., \& Mészáros, P. 2013, ApJ, 776, L39

Katz, J. I. 2016, ApJ, 826, 226

Keane, E. F., Johnston, S., Bhandari, S., et al. 2016, Nature, 530, 453

Kulkarni, S. R., Ofek, E. O., Neill, J. D., Zheng, Z., \& Juric, M. 2014, ApJ, 797, 70

Li, T.-P., \& Ma, Y.-Q. 1983, ApJ, 272, 317

Lorimer, D. R., Bailes, M., McLaughlin, M. A., Narkevic, D. J., \& Crawford, F. 2007, Science, 318, 777

Lyubarsky, Y. 2014, MNRAS, 442, L9

Masui, K., Lin, H.-H., Sievers, J., et al. 2015, Nature, 528, 523

Mingarelli, C. M. F., Levin, J., \& Lazio, T. J. W. 2015, ApJ, 814, L20

Murase, K., Kashiyama, K., \& Meszaros, P. 2016, MNRAS, 461, 1498

Parsons, R. D., \& Hinton, J. A. 2014, Astropart. Phys., 56, 26

Pen, U.-L., \& Connor, L. 2015, ApJ, 807, 179

Petroff, E., Bailes, M., Barr, E. D., et al. 2015, MNRAS, 447, 246

Petroff, E., Barr, E. D., Jameson, A., et al. 2016, PASA, 33, e045

Scholz, P., Spitler, L. G., Hessels, J. W. T., et al. 2016, ApJ, 833, 177

Spitler, L. G., Cordes, J. M., Hessels, J. W. T., et al. 2014, ApJ, 790, 101

Spitler, L. G., Scholz, P., Hessels, J. W. T., et al. 2016, Nature, 531, 202

Thornton, D., Stappers, B., Bailes, M., et al. 2013, Science, 341, 53

Totani, T. 2013, Publ. Astron. Soc. Japan, 65, 12

Vedantham, H. K., Ravi, V., Mooley, K., et al. 2016, ApJ, 824, L9

Williams, P. K. G., \& Berger, E. 2016, ApJ, 821, L22

Zhang, B. 2014, ApJ, 780, L21

Zhang, B. 2016, ApJ, 822, L14

1 Centre for Space Research, North-West University, Potchefstroom 2520, South Africa

2 Universität Hamburg, Institut für Experimentalphysik, Luruper Chaussee 149, 22761 Hamburg, Germany

3 Max-Planck-Institut für Kernphysik, PO Box 103980, 69029 Heidelberg, Germany

4 Dublin Institute for Advanced Studies, 31 Fitzwilliam Place, Dublin 2, Ireland 
H.E.S.S. Collaboration et al.: First limits on the very-high energy gamma-ray afterglow emission of a fast radio burst

5 National Academy of Sciences of the Republic of Armenia, Marshall Baghramian Avenue, 24, 0019 Yerevan, Republic of Armenia

6 Yerevan Physics Institute, 2 Alikhanian Brothers St., 375036 Yerevan, Armenia

7 Institut für Physik, Humboldt-Universität zu Berlin, Newtonstr. 15, 12489 Berlin, Germany

${ }^{8}$ University of Namibia, Department of Physics, Private Bag 13301, Windhoek, Namibia

9 GRAPPA, Anton Pannekoek Institute for Astronomy, University of Amsterdam, Science Park 904, 1098 XH Amsterdam, The Netherlands

10 Department of Physics and Electrical Engineering, Linnaeus University, 35195 Växjö, Sweden

11 Institut für Theoretische Physik, Lehrstuhl IV: Weltraum und Astrophysik, Ruhr-Universität Bochum, 44780 Bochum, Germany

12 GRAPPA, Anton Pannekoek Institute for Astronomy and Institute of High-Energy Physics, University of Amsterdam, Science Park 904, 1098 XH Amsterdam, The Netherlands

13 Institut für Astro- und Teilchenphysik, Leopold-FranzensUniversität Innsbruck, 6020 Innsbruck, Austria

14 School of Physical Sciences, University of Adelaide, Adelaide 5005, Australia

15 LUTH, Observatoire de Paris, PSL Research University, CNRS, Université Paris Diderot, 5 place Jules Janssen, 92190 Meudon, France

16 Sorbonne Universités, UPMC Université Paris 06, Université Paris Diderot, Sorbonne Paris Cité, CNRS, Laboratoire de Physique Nucléaire et de Hautes Énergies (LPNHE), 4 place Jussieu, 75252 Paris Cedex 5, France

17 Laboratoire Univers et Particules de Montpellier, Université Montpellier, CNRS/IN2P3, CC 72, Place Eugène Bataillon, 34095 Montpellier Cedex 5, France

18 DSM/Irfu, CEA Saclay, 91191 Gif-Sur-Yvette Cedex, France

19 Astronomical Observatory, The University of Warsaw, Al. Ujazdowskie 4, 00-478 Warsaw, Poland

20 Aix-Marseille Université, CNRS/IN2P3, CPPM UMR 7346, 13288 Marseille, France

21 Instytut Fizyki Jạdrowej PAN, ul. Radzikowskiego 152, 31-342 Kraków, Poland

22 School of Physics, University of the Witwatersrand, 1 Jan Smuts Avenue, Braamfontein, Johannesburg 2050, South Africa

23 Laboratoire d'Annecy-le-Vieux de Physique des Particules, Université Savoie Mont-Blanc, CNRS/IN2P3, 74941 Annecy-le-Vieux, France

${ }^{24}$ Landessternwarte, Universität Heidelberg, Königstuhl, 69117 Heidelberg, Germany

25 Université Bordeaux, CNRS/IN2P3, Centre d'Études Nucléaires de Bordeaux Gradignan, 33175 Gradignan, France
${ }^{26}$ Oskar Klein Centre, Department of Physics, Stockholm University, Albanova University Center, 10691 Stockholm, Sweden

27 Institut für Astronomie und Astrophysik, Universität Tübingen, Sand 1, 72076 Tübingen, Germany

${ }_{28}$ Laboratoire Leprince-Ringuet, École Polytechnique, CNRS/IN2P3, 91128 Palaiseau, France

29 APC, AstroParticule et Cosmologie, Université Paris Diderot, CNRS/IN2P3, CEA/Irfu, Observatoire de Paris, Sorbonne Paris Cité, 10 rue Alice Domon et Léonie Duquet, 75205 Paris Cedex 13, France

30 Univ. Grenoble Alpes; CNRS, IPAG, 38000 Grenoble, France

31 Department of Physics and Astronomy, The University of Leicester, University Road, Leicester, LE1 7RH, UK

32 Nicolaus Copernicus Astronomical Center, Polish Academy of Sciences, ul. Bartycka 18, 00-716 Warsaw, Poland

33 Institut für Physik und Astronomie, Universität Potsdam, Karl-Liebknecht-Strasse 24/25, 14476 Potsdam, Germany

34 Friedrich-Alexander-Universität Erlangen-Nürnberg, Erlangen Centre for Astroparticle Physics, Erwin-Rommel-Str. 1, 91058 Erlangen, Germany

35 DESY, 15738 Zeuthen, Germany

36 Obserwatorium Astronomiczne, Uniwersytet Jagielloński, ul. Orla 171, 30-244 Kraków, Poland

37 Centre for Astronomy, Faculty of Physics, Astronomy and Informatics, Nicolaus Copernicus University, Grudziadzka 5, 87-100 Torun, Poland

38 Department of Physics, University of the Free State, PO Box 339, Bloemfontein 9300, South Africa

39 Heisenberg Fellow (DFG), ITA Universität Heidelberg, Germany

40 GRAPPA, Institute of High-Energy Physics, University of Amsterdam, Science Park 904, 1098 XH Amsterdam, The Netherlands

41 Department of Physics, Rikkyo University, 3-34-1 Nishi-Ikebukuro, Toshima-ku, Tokyo 171-8501, Japan

42 Japan Aerpspace Exploration Agency (JAXA), Institute of Space and Astronautical Science (ISAS), 3-1-1 Yoshinodai, Chuo-ku, Sagamihara, Kanagawa 229-8510, Japan

43 Centre for Astrophysics and Supercomputing, Swinburne University of Technology, Mail H30, PO Box 218, VIC 3122, Australia; ARC Centre of Excellence for All-sky Astrophysics (CAASTRO), USA

44 SKA Organisation, Jodrell Bank Observatory, Cheshire, SK11 9DL, UK; ARC Centre of Excellence for All-sky Astrophysics (CAASTRO), USA

45 ASTRON, The Netherlands Institute for Radio Astronomy, Postbus 2, 7990 AA Dwingeloo, The Netherlands

46 Now at Santa Cruz Institute for Particle Physics and Department of Physics, University of California at Santa Cruz, Santa Cruz, CA 95064, USA 